Supporting Information

\title{
Repolarization of M2 to M1 Macrophages Triggered by Lactate Oxidase Released from Methylcellulose Hydrogel
}

\author{
Zi-Xian Liao, ${ }^{a}$ Yu-Chen Fa, ${ }^{a}$ Ivan M. Kempson, ${ }^{b}$ S.-Ja Tseng*,c,d \\ ${ }^{a}$ Institute of Medical Science and Technology, National Sun Yat-sen University, Kaohsiung, \\ 80424, Taiwan \\ ${ }^{\mathrm{b}}$ Future Industries Institute, University of South Australia, Mawson Lakes, South Australia \\ 5095, Australia \\ ${ }^{\mathrm{c}}$ Graduate Institute of Oncology, National Taiwan University College of Medicine, Taipei \\ 10051, Taiwan \\ ${ }^{\mathrm{d}}$ National Taiwan University YongLin Scholar, National Taiwan University, Taipei, 10672, \\ Taiwan
}

\section{Corresponding Author}

*E-mail: sjatseng@ntu.edu.tw (S.J. Tseng). 

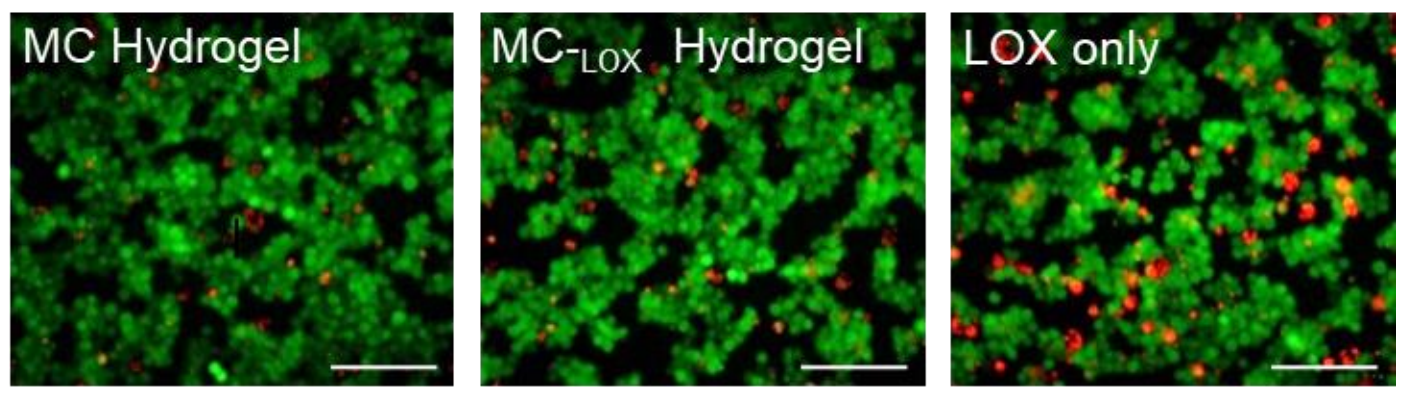

Figure S1. M0 macrophages after exposure to various treatments. After $24 \mathrm{~h}$ incubation, the treated cells were observed using Live/Dead ${ }^{\circledR}$ Stain Kit. Representative confocal images show the red fluorescence (cell death). Bars $=100 \mu \mathrm{m}$. 


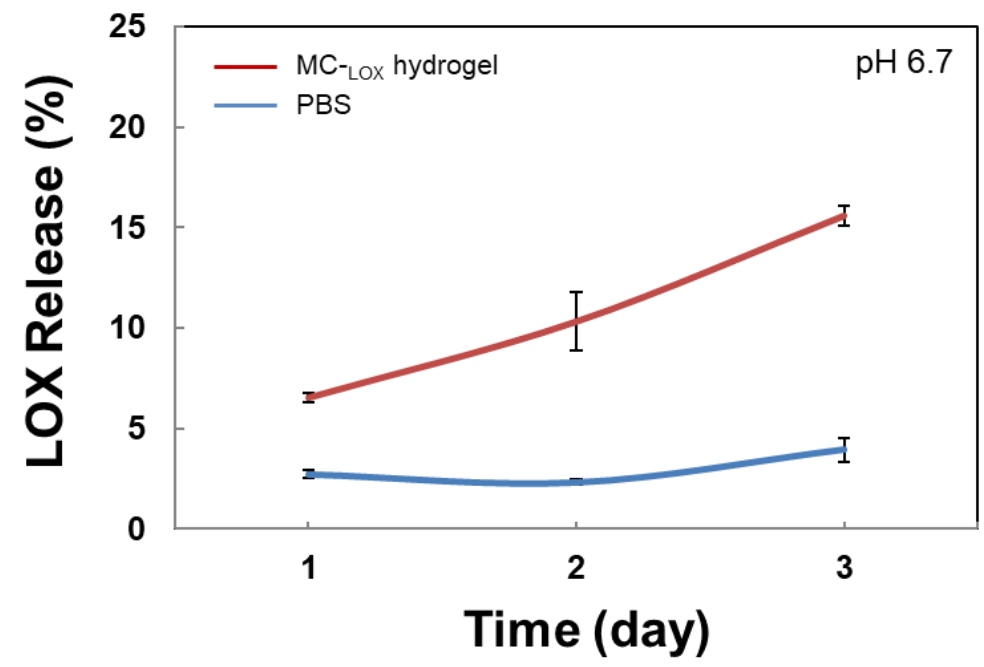

Figure S2. Release of lactate oxidase (LOX) from MC-Lox hydrogels. Measurement of the LOX release. MC-Lox hydrogels were placed in Transwell ${ }^{\mathrm{TM}}$ inserts and incubated in culture medium with $\mathrm{pH} 6.7$ at $37^{\circ} \mathrm{C}$ for 3 days. The bars represent the mean \pm standard deviation $(\mathrm{n}=3)$. 
A

M0 macrophage

$\mathrm{pH} 7.4$
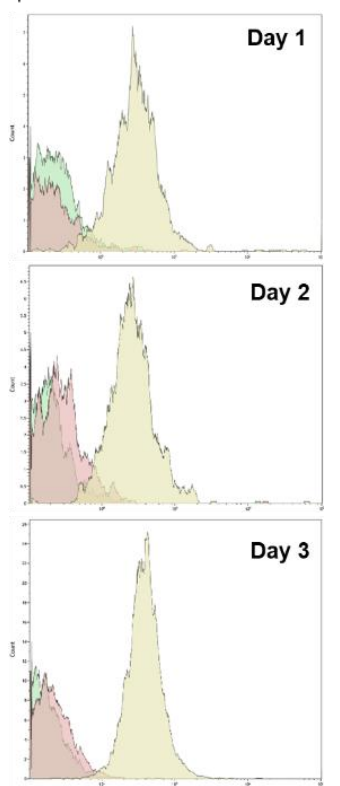

C
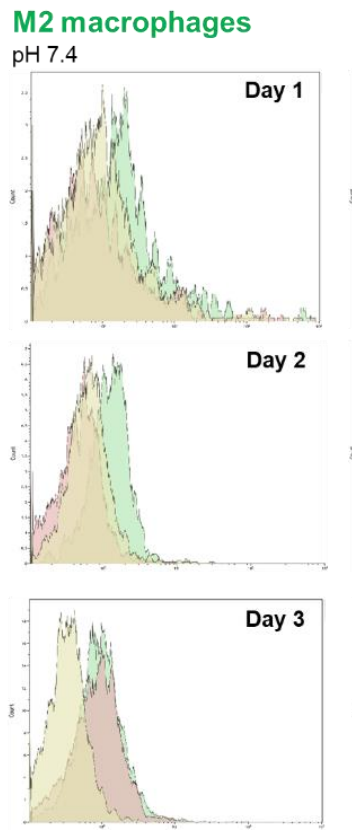

B

M1 macrophages
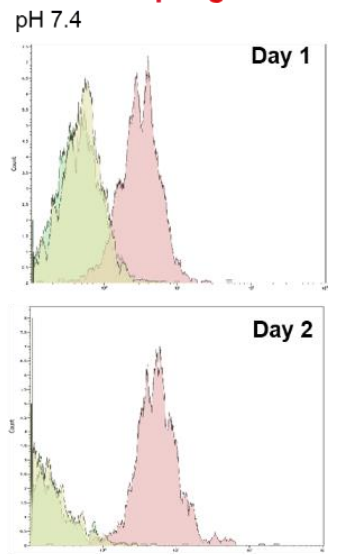

Day 3
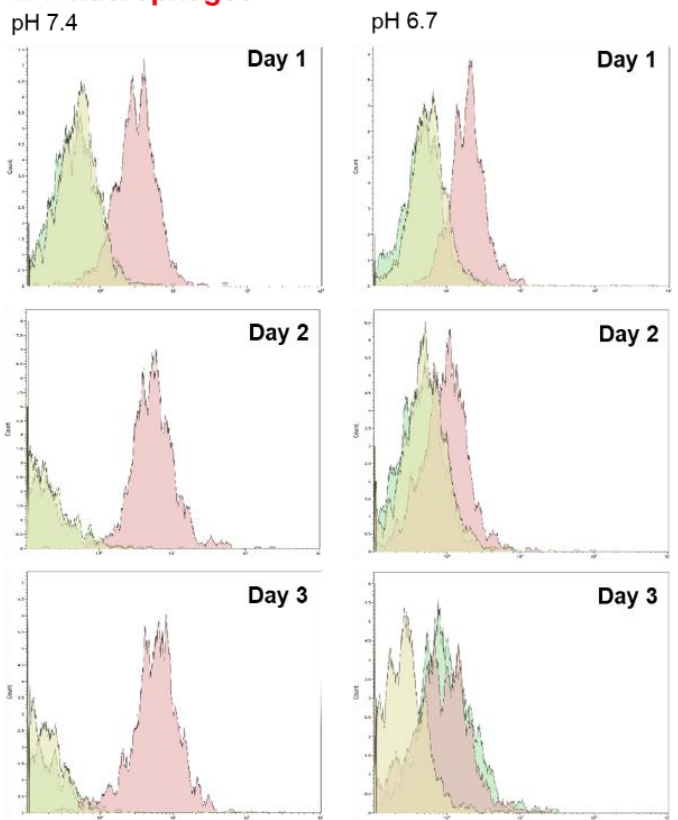

Figure S3. Representative FACS results of M0 (A), M1(B), and M2 (C) macrophages incubated at pH7.4 or 6.7 for 1,2 or 3 days, analyzed by flow cytometry. The markers of F4/80, iNOS, and $\mathrm{CD}^{206}$ identified M0, M1, and M2 macrophages, respectively. The fluorescent signal was developed with Alexa Fluor 633 for F4/80 (yellow), FAM for $\mathrm{CD}^{206}$ (green), or TAMRA for iNOS 
(red). Each fluorescent location was defined by its negative control at the same laser excitation, power, and condition. 

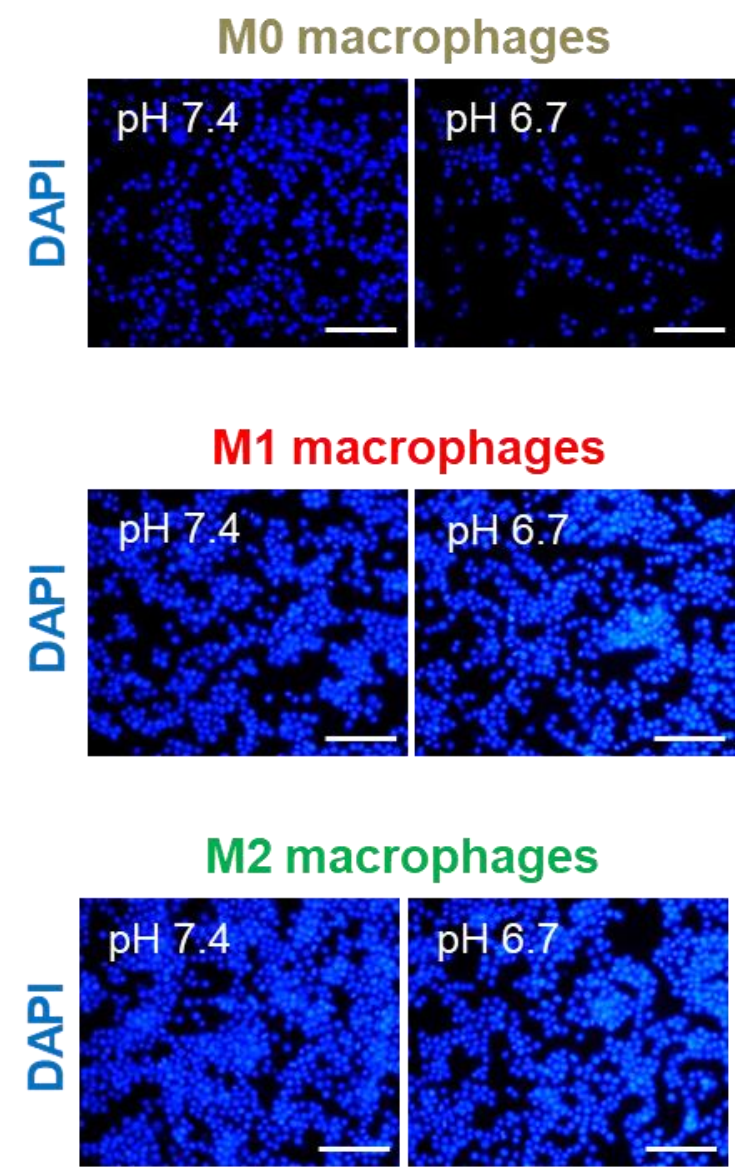

Figure S4. The cells were stained by DAPI to label the cell nuclei of M0, M1, or M2 macrophages incubated at $\mathrm{pH} 7.4$ or 6.7 for 3 days. Bars $=100 \mu \mathrm{m}$. 

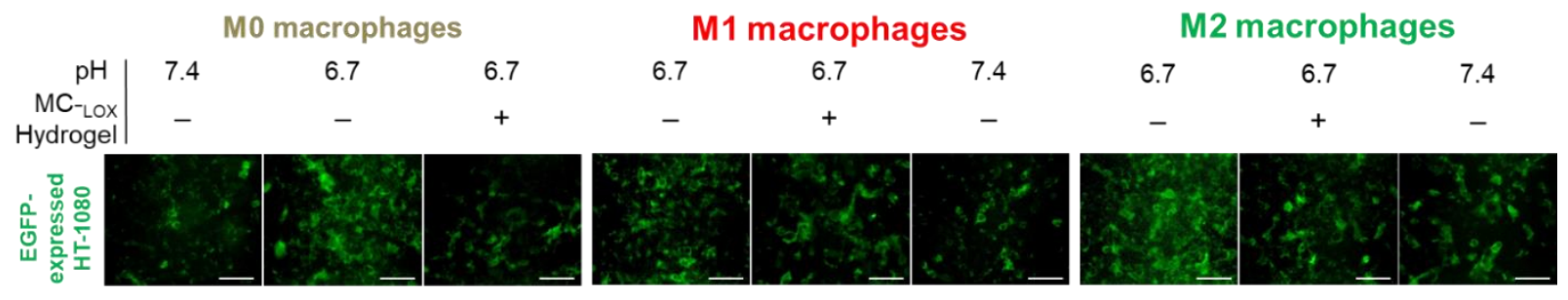

Figure S5. EGFP-expressing HT-1080 cells cocultured with M0, M1, or M2 macrophages incubated w/w or w/o MC-Lox hydrogel at $\mathrm{pH} 7.4$ or 6.7. Representative fluorescent images show EGFP-expressing HT-1080 cells after 3 days incubation. Bars $=100 \mu \mathrm{m}$. 